\title{
Sparse Spectrotemporal Coding of Sounds
}

\author{
David J. Klein \\ Institute of Neuroinformatics, University of Zurich and ETH Zurich, Winterthurerstrasse 190, CH-8057 Zurich, Switzerland \\ Email:djklein@ini.phys.ethz.ch

\section{Peter König} \\ Institute of Neuroinformatics, University of Zurich and ETH Zurich, Winterthurerstrasse 190, CH-8057 Zurich, Switzerland \\ Email: peterk@ini.phys.ethz.ch
}

\author{
Konrad P. Körding \\ Institute of Neurology, University College London, Queen square, London, WC1N 3BG, UK \\ Email: konrad@koerding.de
}

Received 1 May 2002 and in revised form 28 January 2003

\begin{abstract}
Recent studies of biological auditory processing have revealed that sophisticated spectrotemporal analyses are performed by central auditory systems of various animals. The analysis is typically well matched with the statistics of relevant natural sounds, suggesting that it produces an optimal representation of the animal's acoustic biotope. We address this topic using simulated neurons that learn an optimal representation of a speech corpus. As input, the neurons receive a spectrographic representation of sound produced by a peripheral auditory model. The output representation is deemed optimal when the responses of the neurons are maximally sparse. Following optimization, the simulated neurons are similar to real neurons in many respects. Most notably, a given neuron only analyzes the input over a localized region of time and frequency. In addition, multiple subregions either excite or inhibit the neuron, together producing selectivity to spectral and temporal modulation patterns. This suggests that the brain's solution is particularly well suited for coding natural sound; therefore, it may prove useful in the design of new computational methods for processing speech.
\end{abstract}

Keywords and phrases: sparse coding, natural sounds, spectrotemporal receptive fields, spectral representation of speech.

\section{INTRODUCTION}

The brain evolves both overindividual and overevolutionary timescales embedded into the properties of the real world. It thus seems that the properties of any sensory system should be matched with the statistics of the natural stimuli it is typically operating on [1]. This would suggest that the functionality of sensory neurons can be understood in terms of coding optimally for natural stimuli.

This line of inquiry has been fruitful in the visual modality. Many properties of the mammalian visual system can be explained as leading to optimally sparse neural responses in response to pictures of natural scenes. Within this paradigm, it is possible to reproduce the properties of neurons in the lateral geniculate nucleus (LGN) [2] and of simple cells in the primary visual cortex $[3,4,5]$. The term "sparse representation" is often used in these studies to address one of two distinct albeit related meanings: (1) neurons of the population should have significantly distinct functionality in order to avoid redundancy, and (2) the neurons should exhibit sparse activity over time such that their activity level is often close to zero, but is occasionally very high.

A large number of independent component analysis (ICA) (cf. [6,7]) studies also effectively use this principle and demonstrate the computational advantages of such a representation.

It has recently been shown that the spectrotemporal processing exhibited by neurons in the central auditory system, as captured by the spectrotemporal receptive field (STRF) $[8,9,10]$, shares properties with the spatiotemporal processing of the visual system. In particular, neurons in the primary auditory cortex (AI) can also be understood as linear filters acting upon a spectrally and temporally local extent of a peripherally encoded input. In this paper, we demonstrate that these characteristics of the auditory system can also be understood in terms of sparse activity in response to natural input, which in this case is approximated by speech data. Representations that efficiently code for speech data, adequately represented by spectrograms, are also of obvious technical 
interest since the right type of sound representation might be a key to improved recognition of natural language, speech denoising, or speech generation.

\section{METHODS}

\section{Inputs}

Narratives from 29 distinct languages, taken from the language illustrations in part 2 of the handbook of the International Phonetic Association (IPA) (available at http://www2. arts.gla.ac.uk/IPA/sndfiles.html), were preprocessed by simulated peripheral auditory neurons $[11,12]$ using the "NSL Tools" Matlab package (courtesy of the Neural Systems Laboratory, University of Maryland, College Park, available at http://www.isr.umd.edu/CAAR/pubs.html). This peripheral analysis employs constant- $Q$ bandpass frequency analysis similar to that of the mammalian cochlea including highfrequency preemphasis (first-order highpass, corner frequency of $300 \mathrm{~Hz}$ ), followed by nonlinear (sigmoidal) transduction, and half-wave rectification and smoothing (firstorder lowpass, corner frequency of $100 \mathrm{~Hz}$ ) for envelope extraction. As a comparison, we obtained a second dataset by recording audio data from one voluntary human subject (KPK) reading German-language texts, using a standard microphone (Escom) and Cool Edit Pro software (Syntrillium Software, Phoenix, USA) recording mono at 44 kbit, 16 bits precision (Figure 1a). The resulting data can be viewed as spectrograms, which represent the time-dependent spectral energy of sound. An example is shown in Figures 1a and $1 \mathrm{~b}$, which show, respectively, the input and output of the peripheral model to the utterance "Komm sofort nach Hause" ("come home right away" in German).

The spectrograms have 64 points along the tonotopic (spectral) axis, covering a frequency range from 185 to $7246 \mathrm{~Hz}$. This corresponds to a spectral resolution of approximately 12 channels per octave. Temporally, the data is arranged into overlapping blocks of 25 points, each covering 250 milliseconds. One block of data is taken per 10 milliseconds. The data is subjected to a principal component analysis (PCA), and the first 200 components are set to have unit variance (called whitening) and are subsequently used as input vectors $\mathbf{I}(t)$ of length 200 to the optimization algorithm. Thirty thousand subsequent samples are used as input.

\section{Neuron model}

Hundred neurons are simulated, each of which has a weight vector $\mathbf{W}_{i}$ of length 200. The activities $A_{i}$ of the neurons are defined as follows:

$$
A_{i}(t)=\mathfrak{\vartheta}\left(\mathbf{I}(t) \mathbf{W}_{i}\right)
$$

where $\vartheta$ is the heavyside function: $\vartheta(x)=x$ for $x>0$, $\vartheta=0$ otherwise. The neurons are thus characterized by linear threshold properties. The parameters of the simulated neurons are optimized by a fast optimization algorithm, called resilient backprop [13], to maximize the following objective function:

$$
\Psi_{\text {Cauchy }}=\sum_{i}\left\langle\log \left(1+A_{i}^{2}\right)\right\rangle_{\text {all sounds }},
$$

where $\langle *\rangle$ denotes the average over all stimuli and $A_{i}$ is the activity of the neuron. The simulated neurons, furthermore, should not all have the same properties; they should be decorrelated. We thus add a second term

$$
\Psi_{\text {decorr }}=-\sum_{i, j} \operatorname{CC}\left(a_{i}, a_{j}\right)-\sum_{i}\left(1-\operatorname{std}\left(a_{i}\right)\right)^{2},
$$

where CC denotes the coefficient of covariation and std is the standard deviation. The first term biases the neurons to have distinct activity patterns, while the latter term effectively normalizes the standard deviation. The optimization is performed in principal component space (cf. [14]) for the sake of faster computation. This transformation is done using the Matlab princomp routine.

\section{Analysis}

The optimized spectrotemporal analysis performed by the neurons is characterized by the corresponding STRF according to the learned set of optimal weights. Properties of that STRFs are measured in a manner similar to that used in neurophysiological studies. The best frequency (BF) and best time (BT) are defined as the spectral and temporal locations, respectively, of the maximum weight. The $-10 \mathrm{~dB}$ excitatorytuning bandwidth and duration are defined as the spectral and temporal extent of the portion of the STRF within $1 / \sqrt{10}$ of the peak value.

In addition, properties of the two-dimensional Fourier transform of the STRFs are measured. Here, the spectral and temporal filtering of the input signal is quantified by the peak and cutoff $(-10 \mathrm{~dB})$ frequencies of the Fourier transform, along both the spectral and temporal axes. In addition, the extent to which an STRF encodes frequency modulation of a given direction is quantified by a directionality index. This index is computed as the relative power difference of the first two quadrants of the Fourier transform, which lies between -1 and 1 .

It is illuminating to compare the properties of the optimized network with the statistics of the employed stimulus ensemble. The output of the peripheral auditory processing was thus subjected to a multiscale two-dimensional wavelet (second-derivative Gaussian) analysis [15], from which the energy distribution of temporal and spectral modulations, as a function of tonotopic position, was computed.

\section{RESULTS}

\subsection{Data properties}

The first several principal components of the speech data are shown in Figure 1c. It is evident that those components that represent much of the variance change slowly in spectrum and in time. Thus, using only the first principal components 


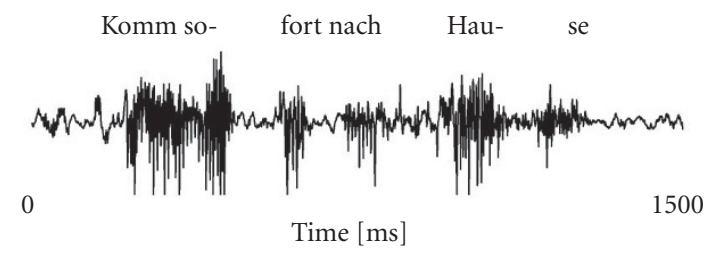

(a)

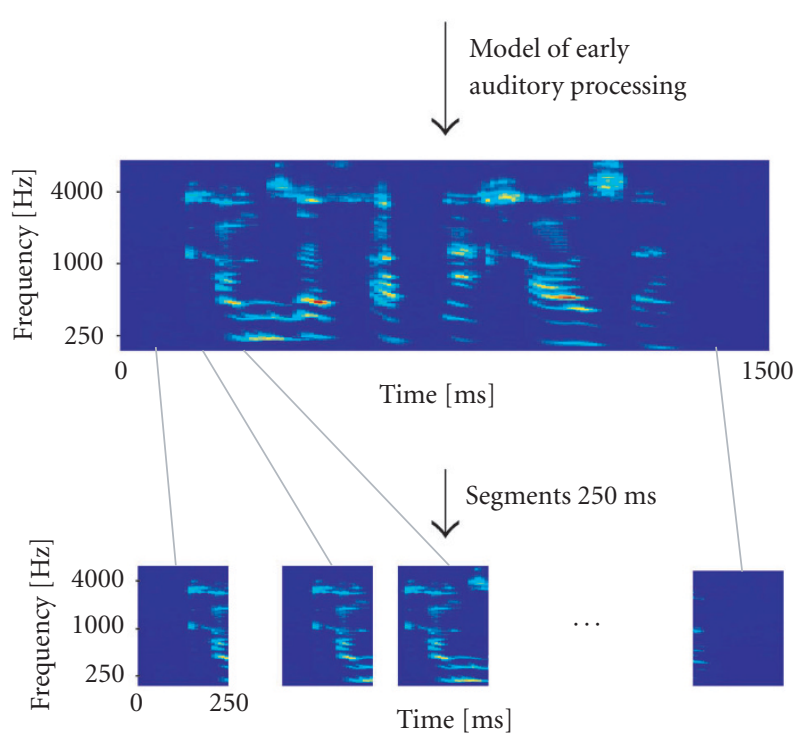

(b)
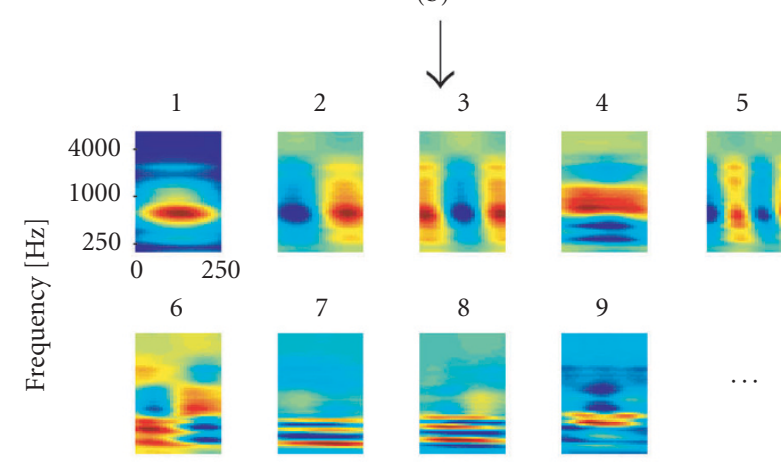

Time $[\mathrm{ms}]$

(c)

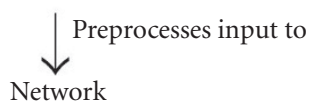

Figure 1: Methods. (a) Recorded speech data are shown as raw waveforms. (b) This data is input to a model of the auditory system's early stages resulting in a spectrogram, where the strength of the activity is color-coded. These spectrograms are subsequently cut into overlapping pieces of length 250 milliseconds for each. The principal components of this PCA are shown in (c), color-coded in a scale where blue represents small values and red represents large values.

for learning effectively low-passes the stimuli; however, the first 200 components that are used for learning account for more than $90 \%$ of the total variance of the data. We previously reported that the form of the lowest components is also robust to changes of the spectrotemporal resolution of the peripheral auditory model [16].
Particularly conspicuous are the fine-scale spectral features that are often evident at lower frequencies. These arise from the low-frequency harmonics that are important for conveying the pitch of voiced speech. At frequencies higher than $1-2 \mathrm{kHz}$, comparatively broader-scale spectral features, corresponding primarily to speech formants, are evident. 


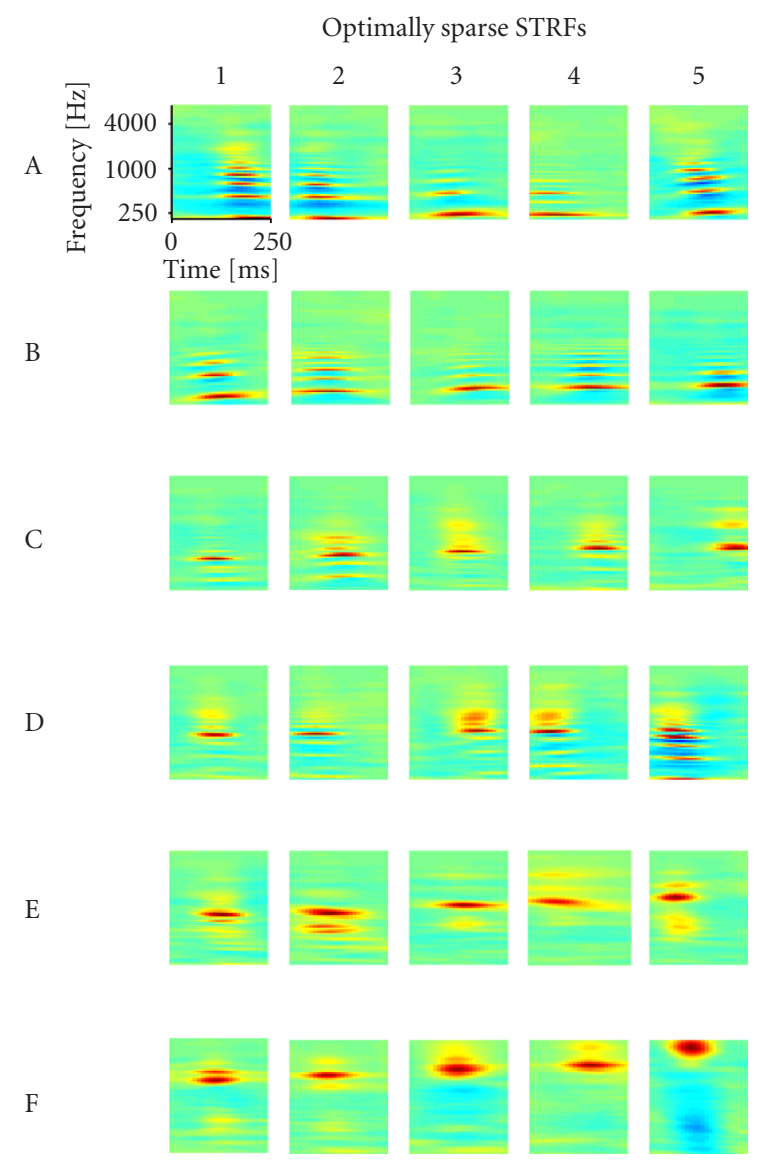

FIGURE 2: Results from optimising sparseness. Representative colorcoded STRFs of the neurons are shown. The same STRF is often seen at different delays. In this case only the one with most energy in the middle of the STRF is shown.

\subsection{Receptive field properties}

The employed IPA speech database contains both male and female speakers from a variety of languages. Representativelearned filters for optimal spectrotemporal encoding of this dataset are illustrated in Figure 2. Here, STRFs of linearthreshold neurons were obtained by optimizing the sparseness of the neurons' stimulus-evoked activity.

A number of important properties can be observed for these STRFs. First of all, the STRFs are typically welllocalized in time and in spectrum. Secondly, many of the STRFs exhibit multiple peaks and troughs in the form of dampened oscillations along the spectral and temporal axes. Finally, a range of frequency modulations are seen in the STRFs; these are evidenced by oblique orientations of the STRFs (e.g., see neurons B1 and E4). It should be noted that all these three properties are also exhibited in STRFs obtained from neurophysiological experiments.

The specific manifestation of these three properties however varies from neuron to neuron. For instance, different STRFs are centered on different regions of the spectrotemporal plane and have different spectral and temporal extents. The distribution of the position and shape parameters for the entire population of STRFs is shown in Figure 3a. For clarity, the sizes of the iconified STRFs have been reduced to $1 / 8$ of their actual values.

In addition, the frequency of the spectral and temporal oscillations exhibited in the STRFs and the amount of dampening varies across the population, as shown in Figures $3 \mathrm{~b}$ and $3 \mathrm{c}$. In the Fourier domain, these two properties conspire to produce selectivity for specific modulations along the temporal or the spectral axis. Figure $3 \mathrm{~b}$ quantifies this selectivity for the spectral domain and shows how the spectral characteristics of the Fourier transform vary as a function of the tonotopic position of the STRF. For about half of the STRFs, the peak of the Fourier transform lies between about 0 and 1 cycle per octave (c/o), and the upper cutoff lies between 1 and $3 \mathrm{c} / \mathrm{o}$. A radically different strategy is seen at low frequencies. Here, many neurons pass significantly higher spectral modulations, ranging from 2 to $6 \mathrm{c} / \mathrm{o}$. This is most likely due to the presence of low-frequency high-scale harmonic patterns in the speech input. Indeed, Figure $3 \mathrm{~d}$ shows that the stimulus itself contains a high proportion of high-scale energy at low frequencies.

The situation is somewhat different in the temporal domain. In general, the STRFs are highly damped in the temporal direction, producing temporal modulation selectivity that is generally lowpass. This fact is evident in Figure 3e which shows that the peak of the Fourier transform never rises above $2 \mathrm{~Hz}$; furthermore, the cutoff frequency is inversely correlated with the temporal width of the STRF and never rises above $10 \mathrm{~Hz}$. Nevertheless, the range of temporal frequencies present in the STRFs is largely consistent with the average temporal composition of the speech input which has a majority of its power concentrated below $10 \mathrm{~Hz}$ (Figure 3f).

Finally, different STRFs exhibit different degrees of frequency modulation, as quantified by the direction selectivity index (Figure $3 \mathrm{~g}$ ). This index is -1 if an STRF exhibits solely positive-going frequency modulations, is 1 for solely negative-going frequency modulations, and is 0 for equal amounts of positive and negative frequency modulation. The population distribution is unimodal and peaks at an index of 0 , which is similar to that obtained from neurophysiological experiments [8].

A number of important properties are shared between these STRFs and those of real neurons. The extent of temporal localization (100-200 milliseconds) and spectral localization (0.5-3 octaves) of the learned STRFs are highly comparable to real STRFs obtained from primary auditory cortex of mammals. This applies also to the range of spectral $(<2 \mathrm{c} / \mathrm{o})$ and temporal $(<10 \mathrm{~Hz})$ modulations represented by the simulated STRFs. There are two notable differences between the simulated and actual STRFs obtained from animals. First, most biological STRFs exhibit bandpass temporal processing, whereas the simulated STRFs are primarily lowpass. Secondly, there exists a large group of artificial neurons that directly encode the fine-scale, multiple-peaked spectral features of pitch; such STRFs have not yet been observed in the auditory system of animals. 


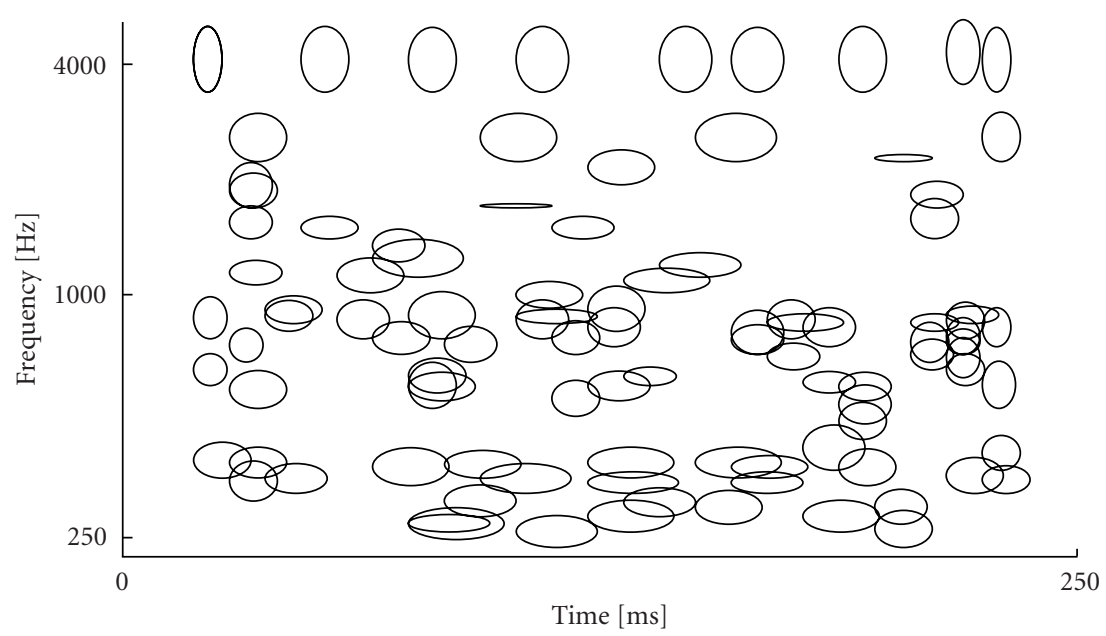

(a)

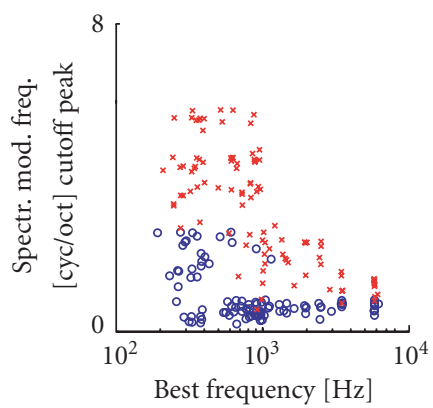

(b)

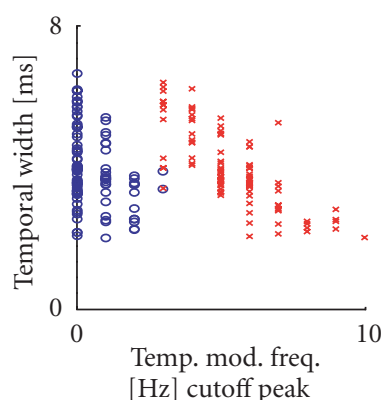

(e)

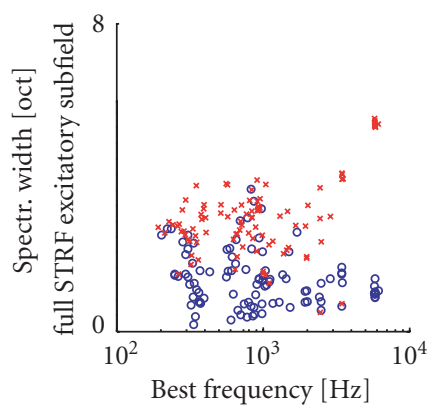

(c)

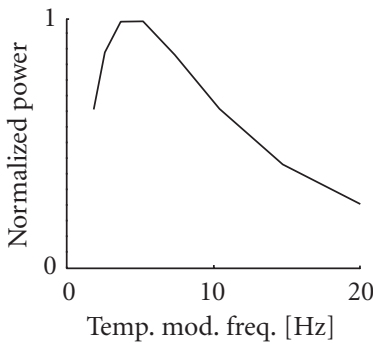

(f)

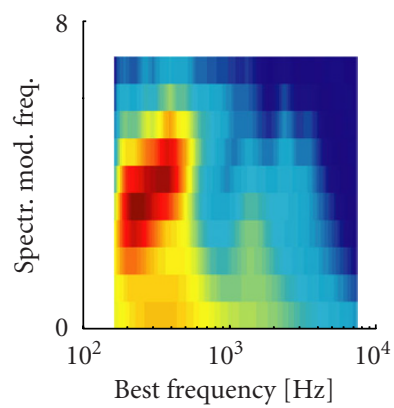

(d)

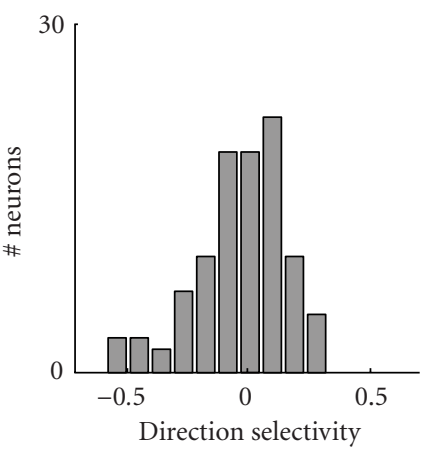

(g)

FIgURE 3: Quantitative properties. (a) The time-frequency tiling properties of the neurons are shown. The center of each ellipse represents the BF and BT of a neuron. The vertical and horizontal extent of the ellipse represents $1 / 8$ of the width of the STRF in the direction of time and spectrum. The factor of $1 / 8$ was introduced to improve visibility of the data. (b) Both the peak (blue) and the high-frequency cutoff (red) of the frequency of spectral modulation are plotted as a function of the best frequency of the neuron. (c) The spectral width of the STRF (red) of the excitatory subregion (blue) is plotted as a function of the BF of the neuron. (d) The IPA stimuli are analyzed in the Fourier domain. The mean of the absolute values of the wavelet transforms of spectrograms taken from the IPA database are shown. (e) Both the peak (blue) and the high-frequency cutoff (red) of the frequency of temporal modulation are plotted as a function of the temporal width of the neuron. (f) The normalized power is shown as a function of the temporal modulation frequency. (g) The index for direction selectivity is calculated and its histogram is shown.

\subsection{Dependence on the neuron model}

The current study has so far focused upon STRFs which produce optimally sparse activity in linear-threshold neu- rons. We used such properties to mimic the properties of real neurons that cannot exhibit negative firing rates. Most ICA studies, however, use purely linear neurons as we did 
in previous simulations [16]. In order to compare the results, we repeated the current simulation using purely linear neurons. Representative results are shown in Figure 4a. Qualitatively speaking, the major features of the neurons' STRFs remain conserved. However, the spectral and temporal extents of the STRFs are typically somewhat larger, and, furthermore, the presence of negative deflections is somewhat reduced. The combination of these two properties produce a spectrotemporal analysis that is more restricted to low spectral and temporal modulation frequencies.

\subsection{Dependence on the dataset}

For the sake of comparison, the simulations with linearthreshold neurons were repeated with a different speech input, this time obtained from a single speaker (see Section 2). Sparseness requires that the neurons should occasionally have very high activity while having small activities most of the time. If we only have one speaker, they can thus be expected to distinguish specific elements of the speaker's speech rather than distinguishing utterances from different speakers. The results are likely not to generalize well to utterances of other speakers while being optimal for utterances of the speaker. Figure $4 \mathrm{~b}$ shows the learned receptive fields. Far more of the neurons code for changes of the pitch of the sounds. A number of them even code for compound features (e.g., neuron D4) where some frequencies rise while others fall. Thus the dataset used clearly influences the data and, therefore, must be carefully considered.

\subsection{Dependence on the number of principal components used}

The number of principal components used to encode the input potentially has some influence on the resulting receptive fields. Low-order principal components tend to be of lower frequency than higher frequency components, and so using fewer principal components has an effect similar to selectively reducing the resolution of the peripheral spectrotemporal analysis. To test the influence of this effective smoothing, the simulations were repeated using reduced numbers of principal components. Even if only 25 components are used (Figure 5), the typical STRF properties described above still apply. This includes even the encoding of low-frequency finescale spectral features due to voice pitch. Thus the number of principal components only has a limited influence on the resulting STRFs.

\section{DISCUSSION}

When analyzing the computational properties of neural systems, it is of central importance to have a thorough understanding of the input representation. A number of other theoretical studies of optimal auditory processing have used raw acoustic-pressure waveforms as input representation. For example, Bell [17] studied the learning of auditory temporal filters using ICA, and Lewicki and Sejnowski [18] showed that using overcomplete representations can significantly

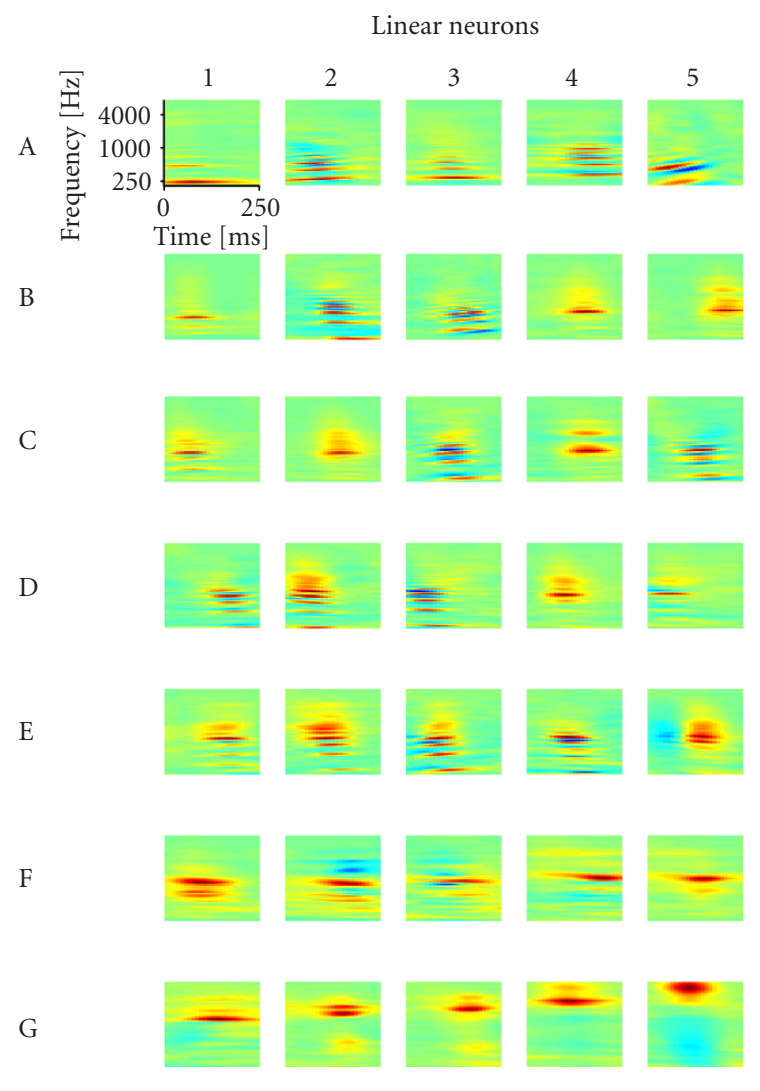

(a)

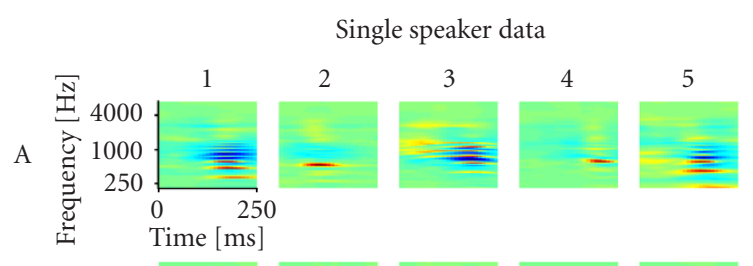

B
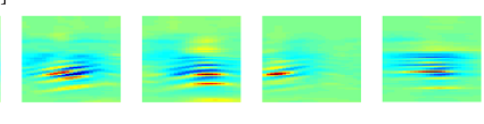

C
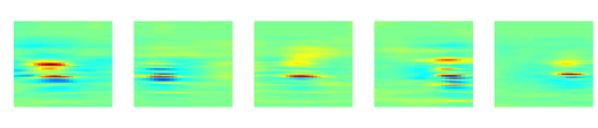

$\mathrm{D}$
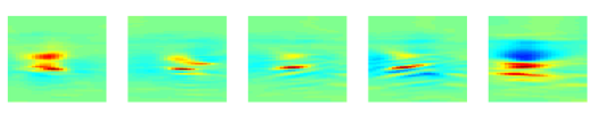

E
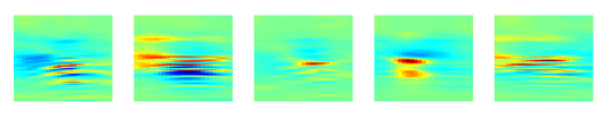

F
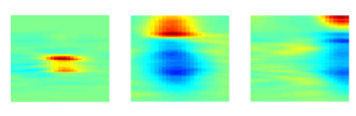

(b)

Figure 4: Dependence on the neuron model and the dataset. (a) The representative STRFs of linear neurons are shown. (b) The representative STRFs of neurons that are optimized on data from a single speaker are shown. 


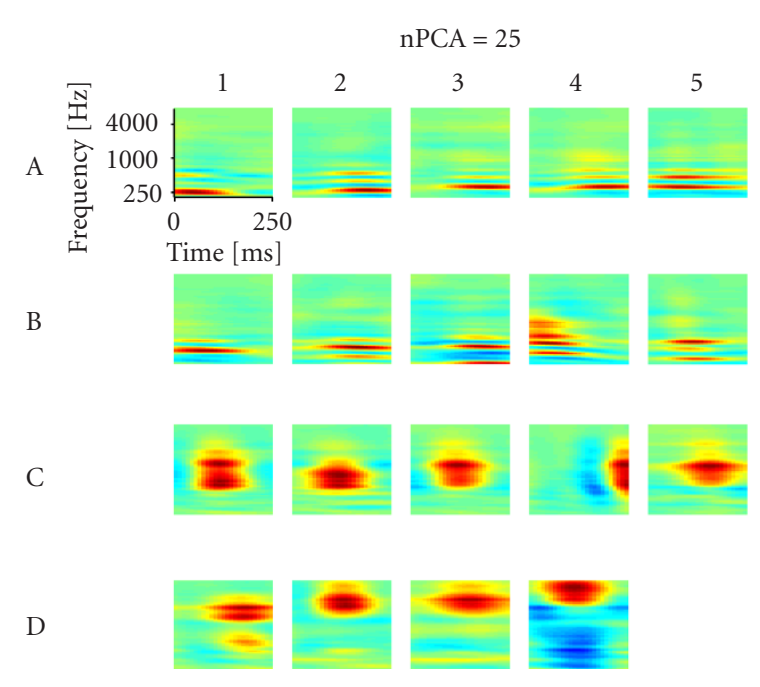

(a)

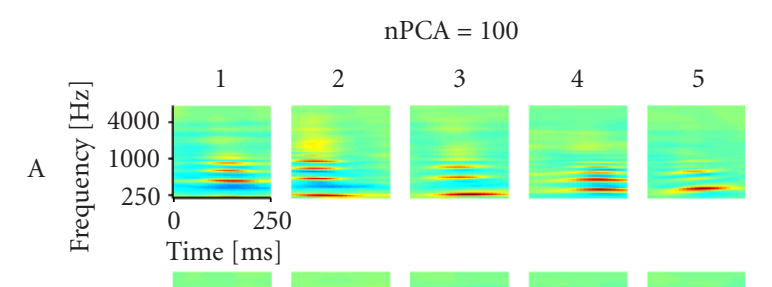

B
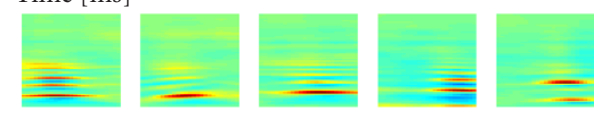

C
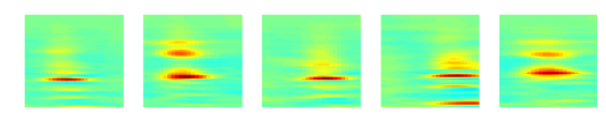

$\mathrm{D}$
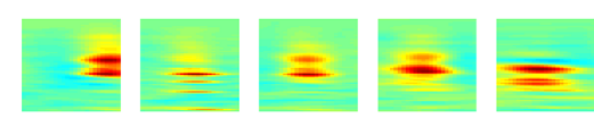

$\mathrm{E}$
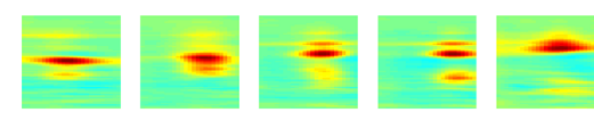

F
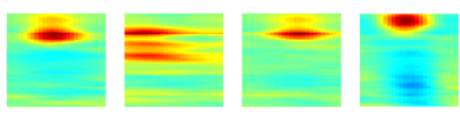

(b)

FIGURE 5: Dependence on the number of principal components. The representative STRFs are shown for a simulation where a smaller number of principal components were used. (a) shows the results from using 25 components and (b) shows the results from using 100 components.

improve the model quality. Using a balanced dataset taken from various sound sources, it was possible to approximately reproduce the spectral analysis performed by the peripheral auditory system [19].
How do central auditory neurons combine the features already encoded by more peripheral neurons? Recent neurophysiological studies in various species have shown that the central auditory system jointly processes the spectral and temporal characteristics of sound in a dazzling variety of ways $[8,9,20,21,22]$. The current study strives to provide a method for speculation on the functionality of such processes. In doing so, the raw waveforms are first preprocessed using an auditory peripheral model. This produces a spectrogram-like representation of sound, which is better suited for describing the information-bearing elements of natural sounds such as speech [23]. Furthermore, since central auditory neurons display largely linear processing of the spectrotemporal envelope of sound $[8,24]$, it increases the likelihood that this style of inquiry will be fruitful. In fact, the results obtained from spectrogram inputs exhibit many of the rich properties of real auditory neurons.

We used PCA to preprocess the spectrogram before feeding it into the sparse coding algorithm. The resulting components capture prominent statistical properties of the data. For example, their banded structure at low frequencies reflects the dominant influence of voice pitch. It is possible that this preprocessing will strongly influence the resulting STRFs. However, one can argue against this likelihood on analytical grounds. Assume the number of principal components used is identical to the number of input samples in each spectrogram. The transformation to the principal component space is then an orthonormal matrix of full rank. Because this matrix can be inverted, each local optimum of the method in PCA space is also an optimum to the method in input space. The chosen principal components can thus be expected to be of minor influence on the resulting STRFsprovided that enough principal components are used. Our simulations showed that 100 components, or perhaps even fewer, are enough. Note also that it is possible (albeit numerically very expensive) to perform the simulations without PCA preprocessing, and doing so results in similar STRFs (data not shown).

STRFs in the auditory system show some functional similarity to spatiotemporal receptive fields in the visual system. Many properties of visual neurons have been successfully learned by maximizing sparseness [25]; it has even been possible to quantitatively compare the properties of the visual system with the properties of simulated neurons, (e.g., [4]). This study extends such methods to auditory data. However, while our simulations are able to qualitatively reproduce many important properties of neurons in AI, a thorough quantitative comparison of properties remains an important problem for further research. In doing so, it will first be necessary to assemble a rich database of sounds that accurately represents an animal's acoustic biotope. One must then devise a compact learning mechanism that leads to neuronal properties that closely correspond to those observed experimentally. This might even necessitate the development of novel algorithms for learning nonlinear neuronal properties.

Along with the assumption that the brain learns to sparsely code for natural sounds comes an experimental 
prediction. During an animal's development, one should be able to manipulate the sparseness statistics of the acoustic environment, such that the neurophysiological properties of neurons are altered. For example, raising an animal in an auditory environment where a certain frequency band is sparser than another should enhance the neural representation of the sparse frequencies. Furthermore, one might facilitate auditory learning by making the underlying auditory features more sparse. In fact, the training procedures for children demonstrated in [26] can be interpreted in this framework.

In the visual modality, the sparse representation of images enabled new computational approaches which lead to powerful algorithms for image denoising [7], image compression [27], and preprocessing for image recognition. This suggests that using sparse coding on spectrotemporal data might well lead to better algorithms for the denoising of speech, for the compression of auditory data, and for the recognition of natural language.

\section{ACKNOWLEDGMENTS}

We want to thank the Swiss National Science Foundation (SNF), Swiss Priority Programs (SPP), Collegium Helveticum (KPK), the Swiss National Fonds (PK) Grant no. 3100-51059.97), and the EU AMOUSE project (KPK and PK). We would like to thank the ADA- "the Intelligent Room" Project that is part of EXPO 2002, which partially pays DJK and in part inspired this project. We would like to thank Bruno Olshausen, Tony Bell, and Heather Read for inspiring discussions and technical assistance.

\section{REFERENCES}

[1] H. B. Barlow, "Possible principles underlying the transformations of sensory messages," in Sensory Communication, W. A. Rosenblith, Ed., pp. 217-234, MIT Press, Cambridge, Mass, USA, 1961.

[2] J. J. Atick, "Could information theory provide an ecological theory of sensory processing?", Network: Computation in Neural Systems, vol. 3, no. 2, pp. 213-251, 1992.

[3] B. A. Olshausen and D. J. Field, "Emergence of simple-cell receptive field properties by learning a sparse code for natural images," Nature, vol. 381, no. 6583, pp. 607-609, 1996.

[4] J. H. van Hateren and A. van der Schaaf, "Independent component filters of natural images compared with simple cells in primary visual cortex," Proc. R. Soc. Lond. B, vol. 265, pp. 359-366, November 1998.

[5] A. J. Bell and T. J. Sejnowski, "The "independent components" of natural scenes are edge filters," Vision Research, vol. 37, no. 23, pp. 3327-3338, 1997.

[6] P. Comon, "Independent component analysis," in Proc. Int. Sig. Proc. Workshop on Higher-Order Statistics, J. L. Lacoume, Ed., Chamrousse, France, July 1991.

[7] A. Hyvärinen, "Sparse code shrinkage: Denoising of nongaussian data by maximum likelihood estimation," Neural Computation, vol. 11, no. 7, pp. 1739-1768, 1999.

[8] D. A. Depireux, J. Z. Simon, D. J. Klein, and S. A. Shamma, "Spectro-temporal response field characterization with dy- namic ripples in ferret primary auditory cortex," Journal of Neurophysiology, vol. 85, no. 3, pp. 1220-1234, 2001.

[9] R. C. deCharms, D. T. Blake, and M. M. Merzenich, "Optimizing sound features for cortical neurons," Science, vol. 280, pp. 1439-1444, 1998.

[10] S. A. Shamma, "On the role of space and time in auditory processing," Trends in Cognitive Sciences, vol. 5, no. 8, pp. 340348, 2001.

[11] X. Yang, K. Wang, and S. A. Shamma, "Auditory representations of acoustic signals," IEEE Transactions on Information Theory, vol. 38, no. 2, pp. 824-839, 1992.

[12] K. Wang and S. A. Shamma, "Spectral shape analysis in the central auditory system," IEEE Trans. Speech and Audio Processing, vol. 3, pp. 382-395, 1995.

[13] M. Riedmiller and H. Braun, "A direct adaptive method for faster backpropagation learning: The RPROP algorithm," in Proc. IEEE International Conference on Neural Networks, H. Ruspini, Ed., pp. 586-591, San Francisco, Calif, USA, March 1993.

[14] A. Hyvärinen, "Survey on independent component analysis," Neural Computing Surveys, vol. 2, pp. 94-128, 1999.

[15] T. Chi, Y. Gao, M. C. Guyton, P. Ru, and S. A. Shamma, "Spectro-temporal modulation transfer functions and speech intelligibility," Journal of the Acoustical Society of America, vol. 106, no. 5, pp. 2719-2732, 1999.

[16] K. P. Körding, P. König, and D. J. Klein, "Learning of sparse auditory receptive fields," in Proc. International Joint Conference on Neural Networks, Honolulu, Hawaii, USA, May 2002.

[17] A. J. Bell and T. J. Sejnowski, "Learning the higher-order structure of a natural sound," Network: Computation in Neural Systems, vol. 7, no. 2, pp. 261-266, 1996.

[18] M. S. Lewicki and T. J. Sejnowski, "Learning overcomplete representations," Neural Computation, vol. 12, no. 2, pp. 337365, 2000.

[19] M. S. Lewicki, "Efficient coding of natural sounds," Nature Neuroscience, vol. 5, no. 4, pp. 356-363, 2002.

[20] L. M. Miller, M. A. Escabí, H. L. Read, and C. E. Schreiner, "Spectrotemporal receptive fields in the lemniscal auditory thalamus and cortex," Journal of Neurophysiology, vol. 87, no. 1, pp. 516-527, 2002.

[21] K. Sen, F. E. Theunissen, and A. J. Doupe, "Feature analysis of natural sounds in the songbird auditory forebrain," Journal of Neurophysiology, vol. 86, no. 3, pp. 1445-1458, 2001.

[22] J. J. Eggermont, "Wiener and Volterra analyses applied to the auditory system," Hearing Research, vol. 66, no. 2, pp. 177201, 1993.

[23] L. Cohen, Time-Frequency Analysis, Prentice Hall, Englewood Cliffs, NJ, USA, 1995.

[24] N. Kowalski, D. A. Depireux, and S. A. Shamma, "Analysis of dynamic spectra in ferret primary auditory cortex. II. Prediction of unit responses to arbitrary dynamic spectra," Journal of Neurophysiology, vol. 76, no. 5, pp. 3524-3534, 1996.

[25] B. A. Olshausen, "Sparse codes and spikes," in Probabilistic Models of the Brain: Perception and Neural Function, R. P. N. Rao, B. A. Olshausen, and M. S. Lewicki, Eds., MIT Press, Cambridge, Mass, USA, 2001.

[26] M. M. Merzenich, W. M. Jenkins, P. Johnson, C. Schreiner, S. L. Miller, and P. Tallal, "Temporal processing deficits of language-learning impaired children ameliorated by training," Science, vol. 271, no. 5245, pp. 77-81, 1996.

[27] B. A. Olshausen, P. Sallee, and M.S. Lewicki, "Learning sparse image codes using a wavelet pyramid architecture," in Advances in Neural Information Processing Systems, MIT Press, Cambridge, Mass, USA, 2001. 
David J. Klein was born in Florida (USA) in 1972. His undergraduate and graduate education in electrical engineering was obtained from the Georgia Institute of Technology (Atlanta, Georgia) and the University of Maryland (College Park, Maryland), respectively. $\mathrm{He}$ is currently working as a Visiting Scientist at the Institute of Neuroinformatics (University/ETH Zürich) in Zürich, Switzerland, where he is developing

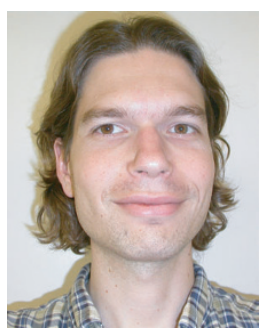
models of auditory cortical function and investigating the usefulness of auditory neuroscientific knowledge for various acoustical signal processing applications, including sound recognition, pitch extraction, and sound localization.

Peter König studied physics and medicine at the University of Bonn, Bonn, Germany. He was with the Department of Neurophysiology at the Max Planck Institute for Brain Research, Frankfurt, Germany, where he received the Habilitation degree in 1990. After working as a Senior Fellow at the Neurosciences Institute in San Diego, Calif, he joined the Institute of Neuroinformatics, Zürich, Switzerland, in 1997. Here, he is us-

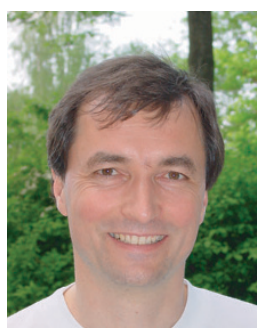
ing experimental and theoretical approaches to study the mammalian visual system, with a particular interest in the processing of natural stimuli.

Konrad P. Körding was born in Darmstadt, Germany, in 1973. He studied Physics in Heidelberg and Zürich. He got his Ph.D. degree from ETH Zürich addressing optimality in the visual system. He worked on biophysical modelling of single cells, oscillatory network dynamics of spiking neurons. More recently, he worked on learning rules that replicate the properties of visual neurons when trained on natural stimuli. His

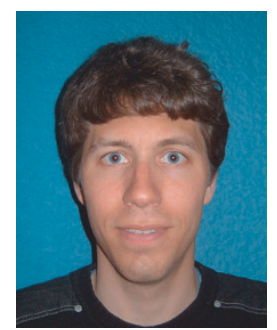
main interests are models of optimal coding in the brain and probabilistic models of the real world. He currently works as a Postdoctor at UCL London and addresses optimality in the motor system. 